\title{
Electronic System for Reactive Power Compensation of Sampled Resistive Load
}

\author{
Milan Stork \\ Dept. of Applied Electronics and \\ Telecommunications/RICE \\ University of West Bohemia \\ Plzen, Czech Republic \\ stork@kae.zcu.cz
}

\author{
Daniel Mayer \\ Dept. of Theory of Electrical \\ Engineering \\ University of West Bohemia \\ Plzen, Czech Republic \\ mayer@kte.zcu.cz
}

\begin{abstract}
Although the mechanism of electric energy flow for nonsinusoidal conditions is well described today, so fare is not yet available a generalized power theory, and theoretical calculations for the design of such devices as active filters or dynamic compensators. Therefore the task of designing compensators for optimize energy transmission with nonlinear timevarying loads in nonsinusoidal regimes is, far from clear. In this paper it will show that even if the voltage is sinusoidal and load is pure resistive but sampled, the load current contains reactive part which can be compensated. The compensation is based on the dissipative systems theory which can be used for calculation of compensation elements (capacitors, inductors) for reactive power compensation. The electronic system for automatic reactive power compensation is also described.
\end{abstract}

Keywords- active power; apparent power; cyclodissipativity; compensation; dissipative systems; nonlinear load; reactive power

\section{INTRODUCTION}

One of the most significant current discussions in electrical engineering is the definition of the reactive power (RP) under nonsinusoidal conditions in nonlinear electric systems. New definitions of RP have been discussed in the last years. Although the mechanism of electric energy flow for nonsinusoidal conditions is well described today, so fare is not yet available a generalized power theory, and theoretical calculations for the design of such devices as active filters or dynamic compensators. Therefore the task of designing compensators for optimize energy transmission with nonlinear time-varying loads in nonsinusoidal regimes is, far from clear. Moreover, in the last time more and more the switching power supplies are used. In this paper it will shown that if the sinusoidal voltage and sampled pure resistive load are used, the load current can contain reactive part which can be compensated by means of simple compensating elements - capacitor or inductor [ 1 -3]

The simplified electrical circuit with sampled resistance load is displayed in Fig. 1. Suppose that

Milan Stork's participation was supported by Department of Applied Electronics and Telecommunications, University of West Bohemia, Plzen and by the Ministry of Education, Youth and Sports of the Czech Republic under the RICE û New Technologies and Concepts for Smart Industrial Systems, project No. LO1607 and by the Internal Grant Agency of University of West Bohemia in Pilsen, Czech Republic the project SGS-2015-002 and GA15-22712S. equation of harmonic source is

$$
v_{s}(t)=\sqrt{2} \cdot A_{1} \cdot \sin \left(\omega_{0} t\right)=\sqrt{2} \cdot 220 \cdot \sin (100 \pi t) \quad[\mathrm{V}]
$$

and load resistor $R=10 \quad[\Omega]$. Switch is sampled (switched ON/OFF) by signal $S(t)$

$$
S(t)=\frac{1+\operatorname{sign}\left[\sin \left(k_{S} \cdot 100 \cdot \pi \cdot t-\alpha\right)\right]}{2}
$$

synchronously with signal $v_{S}$ but with $k_{S}$ time's higher frequency, with phase shift $\alpha$ and width $=0.5$. The example is shown in Fig. 2, where $\alpha=\pi$ and $k_{S}=2$.

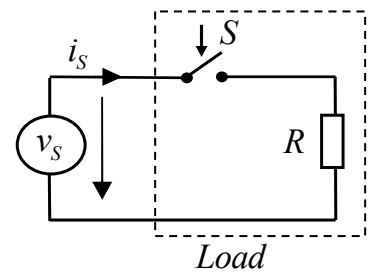

Figure 1. Simplified circuit diagram of sampled resistive load
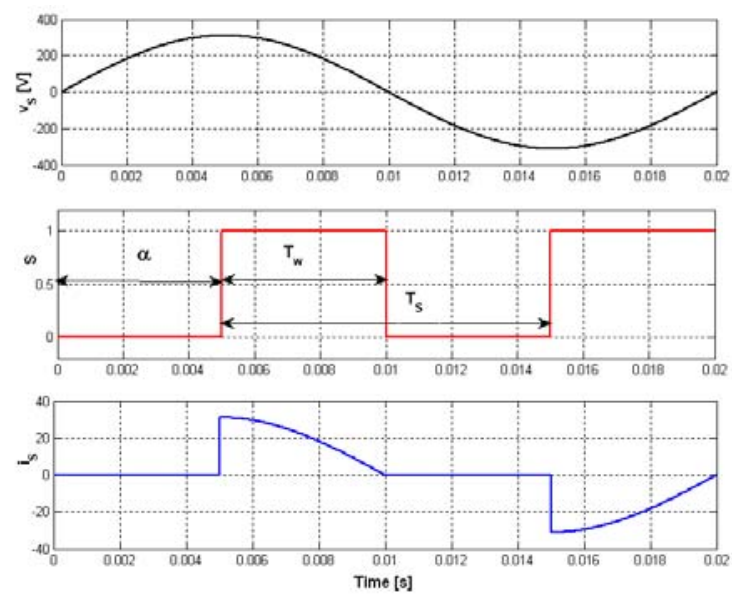

Figure 2. Voltage source, switching signal and current in switched resistive load. Source $v_{S}$, (top), switching signal $S$ (middle), current from source $i_{S}$ (bottom). $T_{S}$ is sampling period, $T_{W}$ is width $\left(T_{W}=T_{S} / 2\right)$ and $\alpha$ is phase shift $\left(T_{D}\right.$ is $T_{S} / 2$ for this example and $\left.k_{S}=2\right)$.

Time evolution of signals for several values of $\alpha$ is presented in Fig. 3. The simplified electrical circuit with sampled resistance load and compensation admittance $Y_{C}$ is shown in Fig. 4. The current from power source is $i_{S}$, compensation current $i_{C O}$ and load current $i$. Principle of RP compensation is described in next part [4].

ISBN 978-80-261-0642-5, (C) University of West Bohemia, 2017 

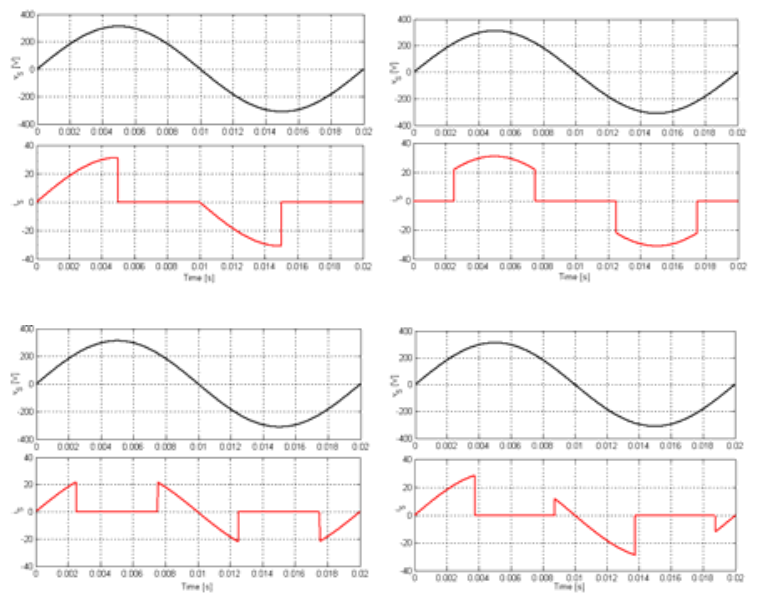

Figure 3. Examples of time evolution of voltage source $v_{S}$ and current $i_{S}$ for different phase shifting of $S(t)$. Left top $\alpha=0$, right top $\alpha=\pi / 2$, left bottom $\alpha=3 \pi / 2$, right bottom $\alpha=7 \pi / 4$.

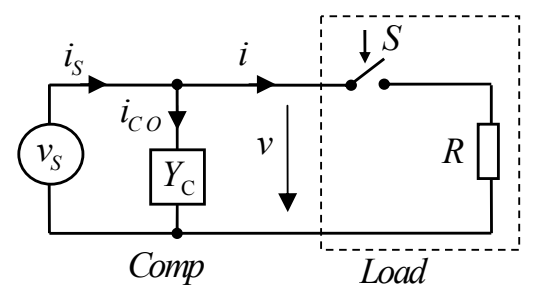

Figure 4. Simplified circuit diagram of sampled resistive load with reactive power compensation by means of compensator $Y_{C}$.

\section{REACTIVE POWER COMPENSATION PRINCIPLE}

The RP compensation of sampling resistive load can be proved by means of spectral decomposition of current $i_{S}$. Suppose time evolution of signals according Fig. 2 (sampling with $\alpha=\pi$ and $k_{S}=2$ ). For nonharmonic current $i_{S}$ the Fourier decomposition was used. The first harmonics of $i_{S}$ (designated as ${ }^{1} i_{S}$ ) and source voltage $\left(v_{S} / 10\right)$ is shown in Fig. 5. From this figure it is clear that phase shift exist between voltage and first spectral component of current, therefore it can be compensated.

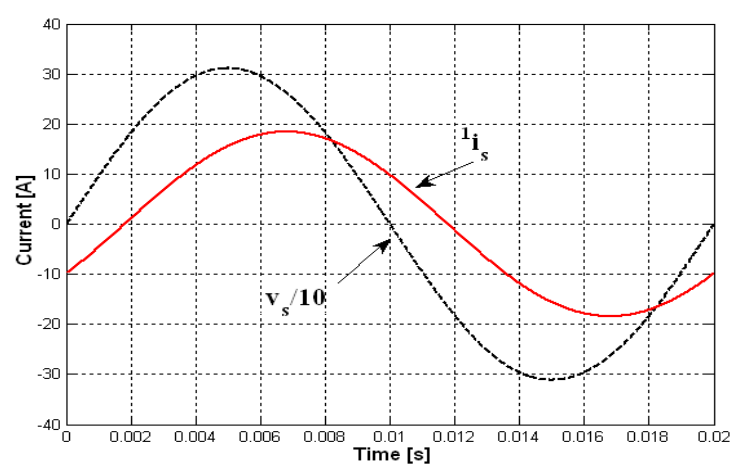

Figure 5. Phase shift between the first spectral component of nonharmonic current $i_{S}$ (according Fig. 2, $\alpha=\pi$ and $k_{S}=2$ ) and voltage source $v_{S} / 10$.

The RP compensation is possible by means of capacitor $C_{C}$, used instead of compensator $Y_{C}$ in Fig. 4. The optimal value of capacitor can be find by optimization, see Fig. 6. From this figure it can be seen, that for optimal compensator the effective current source decreased from 15.5 [A] (without compensation) to 13.9 [A] (with optimal compensation). Voltage and currents after compensation are plots in Fig. 7.

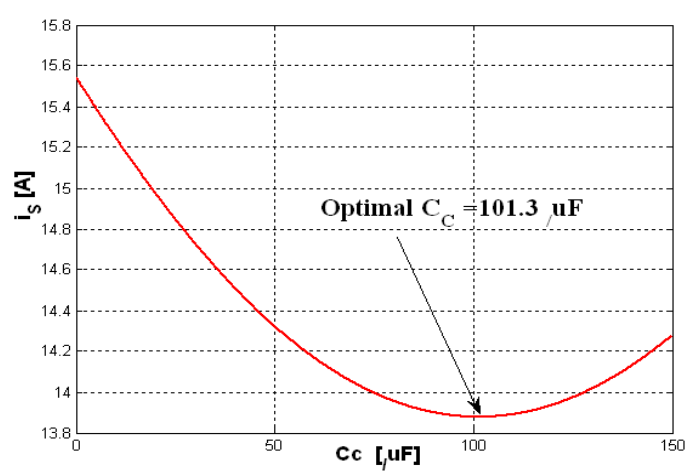

Figure 6. Effective current from source $i_{S}$ versus value of compensating capacitor (for example according Fig. 2).

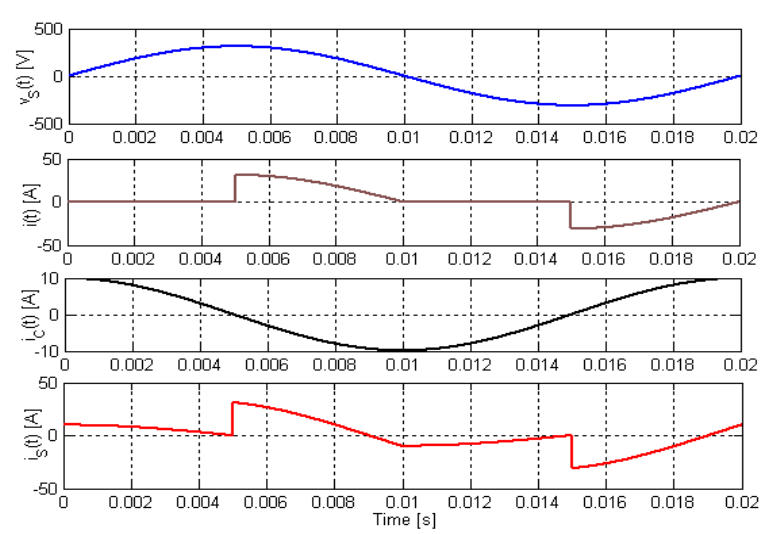

Figure 7. Signals after compensation. From top to bottom: voltage source $v_{S}(t)$, current through the load $i(t)$, current through the compensation capacitor $i_{C O}(t)$, current from the source $i_{S}(t)$.

It must be pointed, that is possible calculate optimal value of compensation by means of mathematical approach [5 - 8]. For any given periodic signal $x(t)$ and $y(t)$ with period $T$ the inner product is defined as

$$
\langle x, y\rangle:=\frac{1}{T} \int_{0}^{T} x(t) y(t) d t
$$

and norm of variable $x(t)$ ( $X$ is rms - root mean square) is

$$
\|x\|=\sqrt{\langle x, x\rangle}:=\sqrt{\frac{1}{T} \int_{0}^{T} x^{2}(t) d t}=X
$$

Let's $x(t)$ and $y(t)$ be differentiable periodic functions with period $T$. Then

$$
\left\langle\frac{d}{d t} x, y\right\rangle=-\left\langle x, \frac{d}{d t} y\right\rangle
$$

and also

$$
\left\langle\int x, y\right\rangle=-\left\langle x, \int y\right\rangle
$$

The active power delivered by the source is defined as 


$$
P:=\left\langle v_{S}, i_{S}\right\rangle=\frac{1}{T} \int_{0}^{T} v_{S}^{\top}(t) i_{S}(t) d t
$$

where $\langle$.$\rangle denotes the inner product. From (7) and the$ Cauchy-Schwarz inequality it follows

$$
P \leq\left\|v_{S}\right\| \cdot\left\|i_{S}\right\|=: S
$$

where the apparent power $S$ is defined. The compensator $Y_{\mathrm{C}}$ is placed in parallel. Also, to avoid power dissipation, $Y_{\mathrm{C}}$ is restricted to be lossless, that is

$$
\left\langle v, i_{C O}\right\rangle=0
$$

where $i_{\mathrm{CO}}$ is compensator current and notice that $v_{\mathrm{S}}=v$ (see Fig.4). From the previous can be derived that the $R P$ compensation problem is mathematically equivalent to the problem of minimization of $\left\|\boldsymbol{i}_{\mathrm{S}}\right\|$ subject to the constraint (9), according

$$
\left\|i_{S}\right\|^{2}=\|i\|^{2}+\left\|i_{C}\right\|^{2}+2\left\langle i_{C}, i\right\rangle
$$

For circuit compensated by capacitor

$$
\left\|i_{S}\right\|^{2}=\left\|i+i_{C O}\right\|^{2}=\|i\|^{2}+\left\|i_{C O}\right\|^{2}+2\left\langle i, i_{C O}\right\rangle
$$

where current $i_{\mathrm{C} 0}$ for compensation capacitor is

$$
i_{C O}(t)=C_{C O} \frac{d v_{S}(t)}{d t}
$$

after substitution and some manipulations

$$
\left\|i_{S}\right\|^{2}=\|i\|^{2}+C_{C O}^{2}\left\|\frac{d v_{S}}{d t}\right\|^{2}+2 C_{C O}\left\langle i, \frac{d v_{S}}{d t}\right\rangle
$$

Minimal value of $i_{\mathrm{S}}$ (or minimum of apparent power $S$ ) can be found for

$$
\frac{d}{d C_{C O}}\left(\|i\|^{2}+C_{C O}^{2}\left\|\frac{d v_{S}}{d t}\right\|^{2}+2 C_{C O}\left\langle i, \frac{d v_{S}}{d t}\right\rangle\right)=0
$$

Optimal value of compensation capacitor is

$$
C_{C O}=-\frac{\left\langle i, \frac{d v_{S}}{d t}\right\rangle}{\left\|\frac{d v_{S}}{d t}\right\|^{2}} \text { or } C_{C O}=\frac{\left\langle\frac{d i}{d t}, v_{S}\right\rangle}{\left\|\frac{d v_{S}}{d t}\right\|^{2}}
$$

where property (5) was used. Optimal value of compensating inductor is

$$
L_{C O}=\frac{-\left\|\int v_{S}\right\|^{2}}{\left\langle i, \int v_{S}\right\rangle} \text { or } L_{C O}=\frac{\left\|\int v_{S}\right\|^{2}}{\left\langle\int i, v_{S}\right\rangle}
$$

where property (6) was used. For compensating element calculation according (15) or (16) is possible the choice what is "better" for calculation. In present example is less complicated use left part of equation (15) to avoid current derivation. Compensation capacitor calculation, according (15) is

$$
C_{C O}=-\frac{\left\langle i, \frac{d v_{S}}{d t}\right\rangle}{\left\|\frac{d v_{S}}{d t}\right\|^{2}}=-\frac{-4.838 \cdot 10^{5}}{(220 \cdot 100 \cdot \pi)^{2}}=101.27 \cdot 10^{-6}
$$
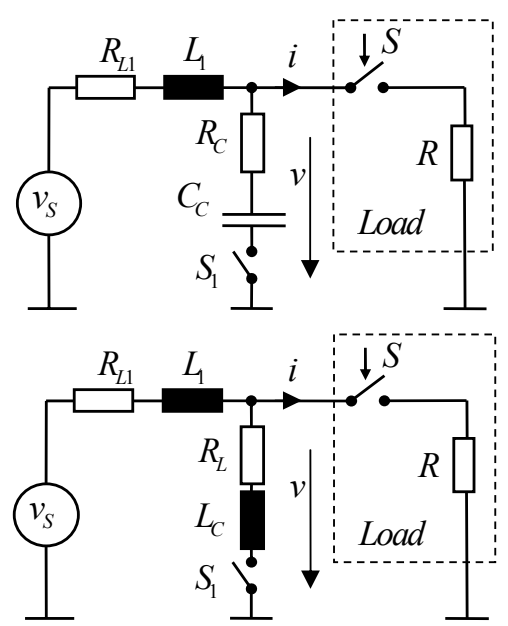

Figure 8. Circuits diagrams for inductive (top) and capacitive (bottom) RP compensation.

\section{RESULTS}

In this part the results of RP compensation are presented. It must be pointed that calculation of compensation inductor, according (16) is also possible, but result is negative (for $\alpha=\pi$ ), which means that compensation by inductor is not real. The types of compensation (capacitive, reactive) depends on phase shift $\alpha$, circuits diagrams are in Fig.8. The results for different values of $\alpha$ are presented in Table 1 (for $\left.k_{\mathrm{S}}=2\right)$. The power factor (PF) is defined as

$$
P F:=P / S \quad[\mathrm{~W}, \mathrm{~V} \cdot \mathrm{A}]
$$

TABLE I. COMPENSATION RESULTS FOR DIFFERENT PHASE OF SAMPLING

$\alpha$ - phase shift of sampling signal, $P F n$ - power factor for noncompensated circuit, $P F C$ - power factor of compensated circuit, Isn - effective current from source for non-compensated circuit, Isc effective current from source for compensated circuit, $C c$ - value of compensation capacitor, $L c$ - value of compensation inductor. If the compensation by capacitor is not possible, value in table is 0 . If the compensation by inductor is not possible, value in table is $\infty$

\begin{tabular}{|c|c|c|c|c|c|c|}
\hline $\boldsymbol{\alpha}$ & PFn & PFc & Isn & Isc & Cc & Lc \\
\hline 0 & 0.708 & 0.793 & 15.57 & 13.91 & 0 & 0.1 \\
\hline 0.314 & 0.773 & 0.841 & 17.02 & 15.66 & 0 & 0.10 \\
\hline 0.628 & 0.829 & 0.872 & 18.234 & 17.33 & 0 & 0.12 \\
\hline 0.943 & 0.87 & 0.891 & 19.138 & 18.69 & 0 & 0.17 \\
\hline 1.257 & 0.9 & 0.902 & 19.712 & 19.59 & 0 & 0.325 \\
\hline 1.571 & 0.904 & 0.904 & 19.9 & 19.89 & 0 & $\infty$ \\
\hline 1.885 & 0.896 & 0.902 & 19.72 & 19.6 & $3.1 \mathrm{E}-05$ & $\infty$ \\
\hline 2.199 & 0.87 & 0.891 & 19.14 & 18.69 & $5.9 \mathrm{E}-05$ & $\infty$ \\
\hline 2.513 & 0.828 & 0.872 & 18.23 & 17.32 & $8.2 \mathrm{E}-05$ & $\infty$ \\
\hline 2.827 & 0.773 & 0.841 & 17.02 & 15.66 & $9.6 \mathrm{E}-05$ & $\infty$ \\
\hline 3.142 & 0.707 & 0.791 & 15.54 & 13.88 & $1.0 \mathrm{E}-04$ & $\infty$ \\
\hline 3.456 & 0.633 & 0.721 & 13.93 & 12.24 & $9.6 \mathrm{E}-05$ & $\infty$ \\
\hline 3.77 & 0.559 & 0.630 & 12.31 & 10.925 & $8.2 \mathrm{E}-05$ & $\infty$ \\
\hline 4.084 & 0.493 & 0.533 & 10.85 & 10.04 & $5.9 \mathrm{E}-05$ & $\infty$ \\
\hline 4.398 & 0.444 & 0.455 & 9.77 & 9.52 & $3.1 \mathrm{E}-05$ & $\infty$ \\
\hline 4.712 & 0.426 & 0.426 & 9.38 & 9.38 & 0 & $\infty$ \\
\hline
\end{tabular}




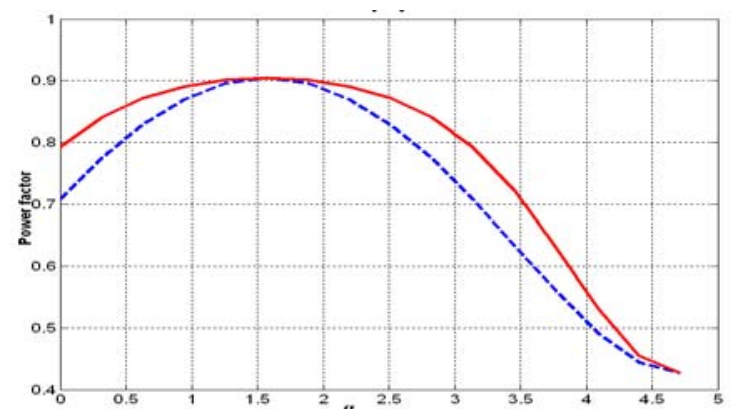

Figure 9. PF for non-compensated circuit (dash-blue) and PF for compensated circuit (solid-red) versus sampling phase $\alpha$.[rad]

$\mathrm{PF}$ for non-compensated and compensated circuit versus sampling phase shift $\alpha$ is shown in Fig. 9. The examples of time diagrams of automatic RP compensation are presented in Fig. 10 and 11. The blocks diagrams of electronic systems for RP compensation are displayed in Fig. 12. Implementation of the control part of system is possible by means of a microcontroller.

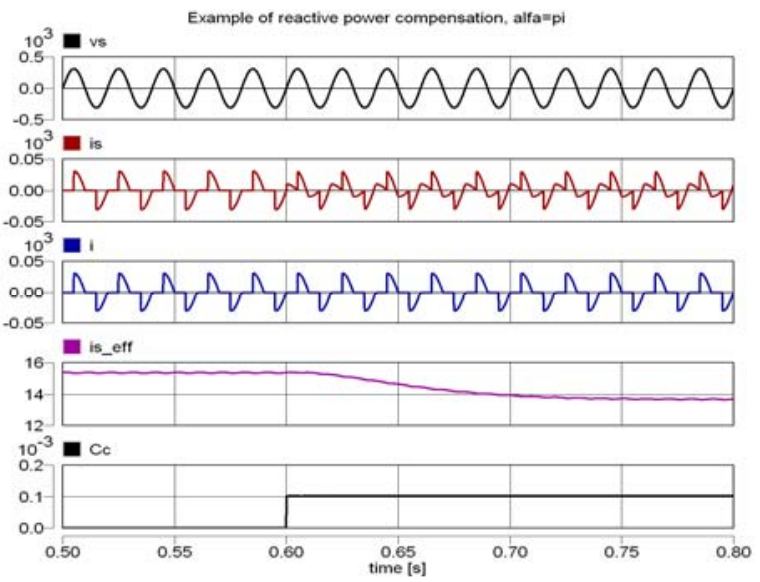

Figure 10. Time diagram - without RP compensation $\left(S_{1}=\mathrm{OFF}\right)$ and with RP compensation $\left(S_{1}=\mathrm{ON}\right)$ for $\left(\alpha=\pi\right.$ and $\left.k_{S}=2\right)$. The switch $S_{1}$ (see Fig. 8) is switched ON for time $>0.6$. From top to bottom: $v_{\mathrm{S}}, i_{\mathrm{S}}$, $i$, is_eff - effective current from source, $C c$ - compensation capacitor.

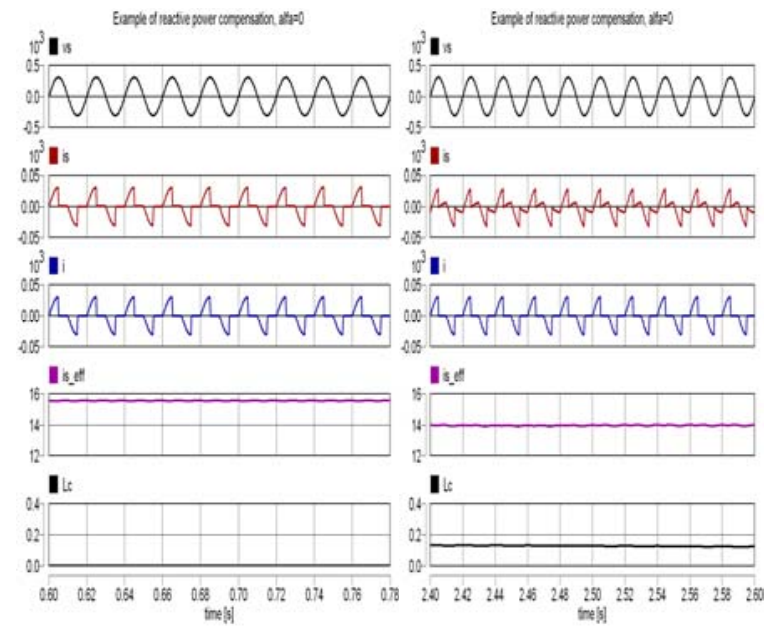

Figure 11. Time diagram - without RP compensation $\left(S_{1}=\mathrm{OFF}\right)$ and with RP compensation $\left(S_{1}=\mathrm{ON}\right)$ for $\left(\alpha=\pi\right.$ and $\left.k_{S}=2\right)$. The switch $S_{1}$ (see Fig. 8) is switched ON for time $>0.8$. From top to bottom: $v_{\mathrm{S}}, i_{\mathrm{S}}$, $i$, is_eff - effective current from source, $L c$ - compensation inductor. The figure is separated on left and right parts because of long transient response after $L c$ is connected in circuit.
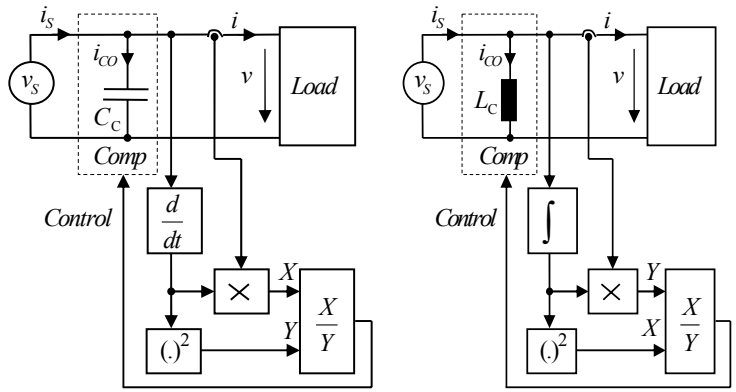

Figure 12. Block diagram of electronic systems for RP compensation - inductive (left) or capacitive (right). This system is suitable for different types of loads (linear, nonlinear or switched load)

\section{CONCLUSION}

In this paper the simple system for RP was presented. On the example of harmonic power source and pure resistive load, but sampled was shown, that for some values of sampling phase shift the RP is generated and can be partly suppressed by means of appropriate passive compensation, therefore PF can be increased [9]. It was also shown that generated RP is inductive or capacitive. Active PFC circuits are based on switchmode converter techniques and are designed to compensate for distortion as well as displacement on the input current waveform. They tend to be significantly more complex than passive approaches, but they are so fare limited for power up to $\mathrm{kW}$.

\section{REFERENCES}

[1] T. S. Key and J.-S. Lai, "IEEE and international harmonic standard impact on power electronic equipment design," in Int. Conf. Industrial Electronics, Control and Instrumentation IECON, pp. 430-436, 1997.

[2] A. Rash, "Power quality and harmonics in the supply network: A look at common practices and standards," in Proc. Mediterranean Electrotechnical Conf. MELECON, pp. 1219$1223,1998$.

[3] IEEE PES Working Group on Nonsinusoidal Situations, "A survey of North American electric utility concerns regarding nonsinusoidal waveforms," IEEE Trans. Power Delivery, vol. 11, pp. 73-78, Jan. 1996.

[4] L. S. Czarnecki,, "Physical reasons of currents RMS value increase in power systems with nonsinusoidal voltage," IEEE Trans. Power Delivery, vol. 8, pp. 437-447, Jan. 1993.

[5] R. Ortega and E. Garcia-Canseco: "Inter-connection and damping assignment passivity-based control: a survey", European Journal of Control: Special Issue on Lagrangian and Hamiltonian Systems, vol. 10, pp. 432-450, December 2004.

[6] A. Astolfi, R. Ortega, and R. Sepulchre: "Stabilization and disturbance attenuation of nonlinear systems using dissipativity theory", European Journal of Control, vol. 8, no. 5, pp. 408-433, 2002.

[7] D. Mayer, J. Hrusak, M. Stork: "On state-space energy based generalization of Brayton-Moser topological approach to electrical network decomposition". Springer, Computing, 2013.

[8] D. Jeltsema: "Modeling and control of nonlinear networks: a power-based perspective", Ph.D. dissertation, Delft University of Technology, The Netherlands, May 2005.

[9] E.Tedeschi, P.Tenti, P.Mattavelli: "Cooperative Operation of Active Power Filters by Instantaneous Complex Power Control", Proc. of the 7th Int. Conf .on Power Electronics and Drive Systems (PEDS 07), Bangkok, Nov 2007. 